\title{
Using AI to Enable Design for Diversity: A Perspective
}

\author{
Fang $\mathrm{Li}^{1}$, Hua Dong ${ }^{2}$ and Long $\mathrm{Liu}^{1}$ \\ ${ }^{1}$ Tongji University, 1239 Siping Road, 200092 Shanghai, P.R. China \\ \{FrankLiFang, LiuLong\}@tongji.edu.cn \\ ${ }^{2}$ Loughborough University, Epinal Way, Loughborough, \\ Leicestershire LE11 3TU, UK \\ H.Dong@lboro.ac.uk
}

\begin{abstract}
Inclusive design focuses on diversity. The contextualized user-sensitive design framework of the interaction system needs to analyze and deal with complex diversity factors, which challenges the traditional design process, tools, and methods. Therefore, new technological progress is needed to provide more innovation potential. The authors point out that the design process of smart products is evolving in response to uncertainty. In the future, diversity-oriented design will tend to allocate design resources and values in an algorithmic way rather than the compromised unity solution. This paper analyzes the limitations and potential of the application of AI technology represented by deep learning in diversityoriented design practice and design research, puts forward the goal and direction of further research, and discusses the critical links of AI-enabled diversity design in interdisciplinary research environment.
\end{abstract}

Keywords: Inclusive Design · Diversity-Oriented Design · Artificial Intelligence

\section{Introduction}

In order to make inclusive design sustainable in the competitive commercial environment, the authors' research focuses on the interdisciplinary study of inclusive design, economics, and technologies. As a design methodology initially driven by values, inclusive design advocates that design should pay more attention to the diversities of people. However, the existing design research generally focuses on how to realize and achieve inclusion in design. The lack of research on the causes and internal driving forces of inclusive design limits its expansion to broader and more universal economic and social fields.

Inspired by Matteo Bianchin and Ann Heylighen's concept of design fairness and justice [1,2], we studied inclusiveness as an additional attribute of products from the perspective of supply/demand distribution in economics and give it economic definition and measurability. We also preliminarily summarized the economic causes of design exclusion, corresponding optimization strategies and sustainable constraints [3].

In view of the diversity of users and contexts in the human-computer interaction (HCI) system, we constructed a contextualized user-sensitive design framework (Fig. 1) based on the analysis of the interaction between the behavior model and various elements. We studied the diversity and impact factors in the HCI system from the two 
primary dimensions of users and contexts. Our research regards the user's demands and capabilities as the functions of the dynamic interaction between the user's characteristics and the environment instead of fixed parameters, and proposes a new user research and analysis tool for user-sensitive design. Based on the contextualized user-sensitive design framework, the methods and system elements of user-sensitive design and contextualized design are studied, as well as the possibility of inclusive design by combining the two [4]. However, if designers want to customize their design to wrap each user's flexible demands and capabilities in different contexts, instead of summarizing diversity as a series of moderate equilibrium points, traditional design processes, tools, and methods are difficult to effectively analyze and deal with complex diversity factors and realize the inclusion of diversity required by contextualized user-sensitive design. Therefore, new technological advances are needed to provide more innovation potential.

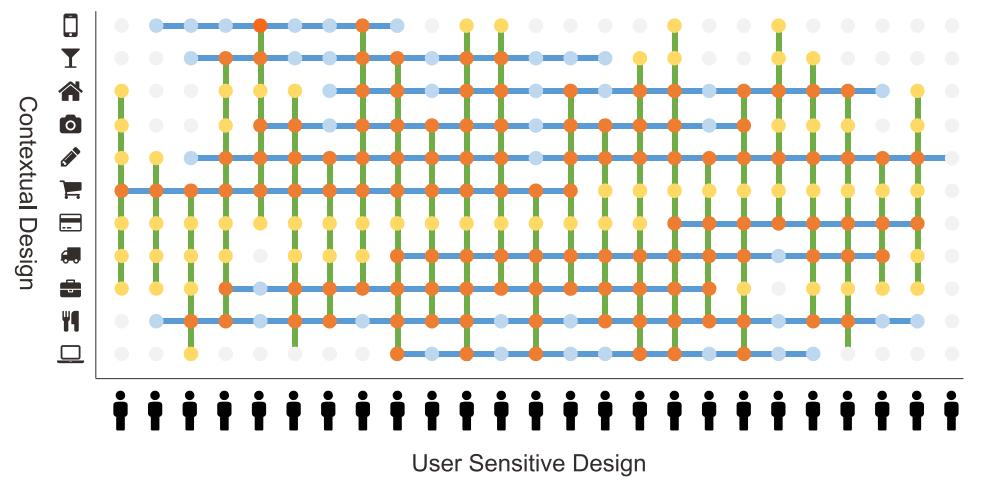

Fig. 1. The basic principle of contextualized user-sensitive design framework. It is to meet the different needs of users with different ability levels in the same scenario, and to ensure that the same user has the same experience in different scenarios [4].

\section{The Shift of Design Process}

Diversity-oriented design echoes the trend of technology, industry, and economy shifting from physical to digital. Traditionally, the physical design paradigm represented by architectural design and industrial design is to seek the best solution under constraints from the preset purpose, thus providing high-quality original design for mass production of high-cost physical products.

The double diamond model (Fig. 2) proposed by the British Design Council summarizes the basic paradigm of the design process described by the traditional design research theory [5]. The two significant diamonds respectively represent two processes. The former process is:

Discover: Start from the initial clear design challenges/objectives and seek to understand various possible causes/constraints through divergent research.

Define: Through analysis, clearly define the requirements and problems, thus determining the corresponding design principles and methods.

Based on the problems identified in the previous process, the latter process then: 
Develop: Try various methods and means to meet the demands and solve the problems.

Delivery: Determine the final output and solutions.

The two diamonds which define the requirements and the solution have both relevance and relative independence. Multiple loop iterations may be required between different nodes or even the entire process. For designers, in specific design practices, many design processes are often directly informed of requirements through design briefing. These demands and problems determined by customers or clients may not have been carefully studied in the Discover and Define stages, or may have been obtained from the professional perspective of product managers or other roles and departments such as marketing. Designers need to decide whether to approve and accept these existing requirement definitions and/or communicate with the corresponding proposer of the requirements. Fortunately, for design researchers, the choice of research topics often has a certain degree of autonomy, and they can start from the research purpose, inject more energy into the Discover and Define stage, to make the research development and output more targeted.

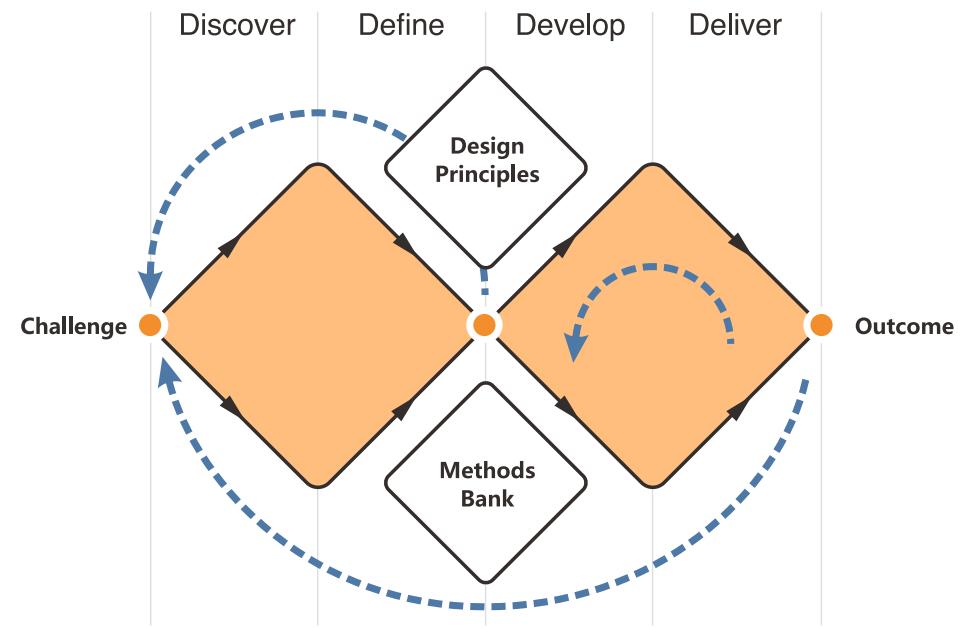

Fig. 2. The British Design Council's double diamond model [5].

However, the virtual design represented by interaction design, experience design, and service design is different. The new paradigm is to establish and match the most suitable solution for diversified needs and individual users under the preset target framework. The reproduction of digital products needs little attention due to little marginal cost, and its supply bottleneck lies in the richness and inclusiveness of design. It aims at establishing a mechanism to generate an uncertain design scheme according to uncertain needs, capabilities, and situations under the guidance of definite objectives (Fig. 3). This process makes it difficult for designers to cope with the challenges of diversity and uncertainty by relying on traditional design methods and tools that seek deterministic solutions. Traditional engineering methods that focus on accuracy and certainty have limitations in helping to solve design problems that are full of uncertainty and complexity. Qualitative research methods in sociology often have to spend much energy on the 
collection and analysis of unstructured data such as field investigation, which is challenging for large-scale applications in commercial design projects that focus on quick response and efficiency. Artificial Intelligence (AI) may be used to help define different problems, and even provide a variety of solutions/outcomes. (Fig. 3)

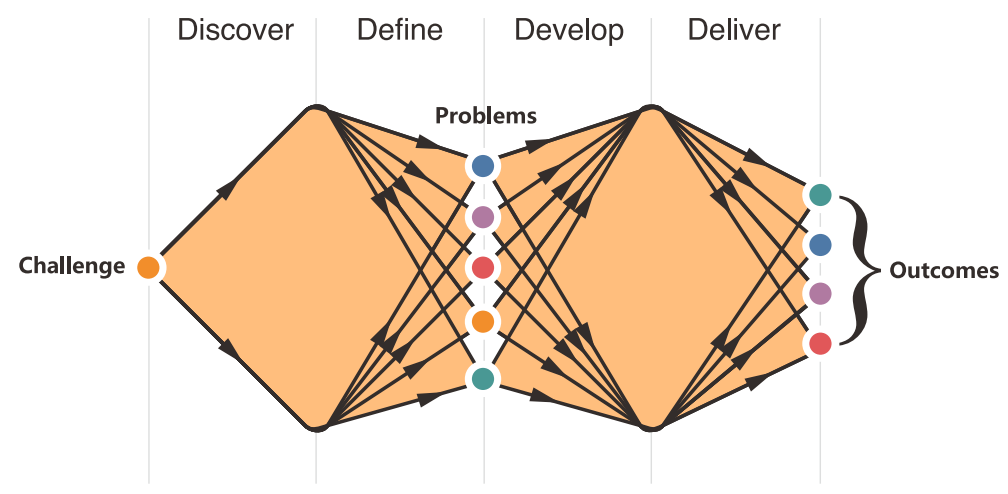

Fig. 3. Evolved design process of diversity-oriented design summarizes differentiated design problems and produces diversified solutions under the same design purpose.

\section{$3 \quad$ Potential of AI}

As low-cost mass customization becomes possible, the design methods to deal with diversity will rely more on inclusivity distribution instead of unity integration. Alternatively, to use the game theory terminology analogy, it is a more mixed strategy instead of a pure strategy. The future diversity-oriented design requires that the design output and even the design itself should become an "algorithm". For specific design purposes, the designer or the designed system needs to establish a mapping relationship between diverse demands and supply. The design process is the exploration and dynamic optimization of the supply potential and its mapping relationship. Designers are supposed to be competent for similar work in a particular field, but the enormous and complex demands may make the diversity-oriented design tasks extremely heavy and make designers struggle to cope with them. Moreover, designers' knowledge structure may not be sufficient to explore the characteristics of different users and different situations in various fields, which requires learning.

In recent years, machine learning technologies such as deep learning have proved their unique advantages in image recognition, NLP (Natural Language Processing), game, and other pattern recognition fields. Although some research has shown that the efficiency of deep learning systems in learning abstract and sophisticated rules is far less than that of human beings [6], sufficient data sets can make up and release the potential of deep learning to a great extent. With the migration and expansion of design research and practice to emerging fields, the types, forms, and accessibility of user and context data have increased significantly. More and more abundant data makes it possible for designers and design researchers to apply data-dependent AI technologies. 
Like image and speech recognition, if AI can learn the features of specific users/scenarios and their corresponding design patterns, and set corresponding design parameters accordingly, it can also provide reference designs for designers or provide alternative solutions for users. This kind of technology will greatly enhance the supply capacity of diversity-oriented design, thus improving the design inclusiveness of diversity requirements.

Existing deep learning and related technologies are still at an early stage in understanding; the results of training based on a large number of training examples are far from the abstract concepts summarized by human beings [7]. Deep learning classifiers are vulnerable to targeted input attacks. Some interferences that do not affect human perception will also significantly reduce the performance of machine-learning trained strategies [8]. Deep learning allows computers to construct complex concepts through simple concepts [9], but deep learning system's generalization and combination ability is still insufficient, so it often requires a huge training example set [10]. The professional experience of designers and design researchers can help optimize the design of deep learning systems for distributed representation of feature abstraction.

On the other hand, from AlphaGo's stunning performance in the Go chess field and DeepMind's open technical framework, it can be seen that the "black box" neural network can achieve performance beyond human level on specific tasks although it cannot directly help us to understand and explain the mysteries of the game more deeply. The "black box" may lead to some divergence of values. Some researchers worry that this state of "knowing what and not knowing why" is a significant shortcoming of machine learning. Lack of interpretability may be very harmful and unacceptable for some applications [11]. However, the Oriental thinking represented by Go chess is used to construct applied knowledge and experience with vague pragmatism before mastering the principle accurately. This kind of thinking is very consistent with the practical characteristics of design activities; it helps to promote new knowledge that is not clear and reliable to play a pioneering role in the design field, which is full of uncertainties. For example, deep learning solves problems from correlation rather than causality, which may not be a problem or shortcoming. We can argue that this makes it possible to explore and obtain feasible and better solutions through deep learning under the control of constraints.

The Deep Convolutional Generative Adversarial Networks (DCGANs) has shown its advantages in unsupervised learning [12]. DeepMind's self-supervised discovery model [13] and machine learning from long-tailed and open-ended distributed data have also made some progress [14]. However, the current deep learning system cannot draw open-ended inferences equivalent to the human-level based on the knowledge of the real-world [7]. Although design activities have a clear purpose, the openness and innovation of design results have always been one of the core values of design. The integration of machine learning technology in the design process needs to give full play to the respective advantages of designers and artificial intelligence, to avoid falling into a design cage of excessively focusing on known requirements [15]. 


\section{$4 \quad$ Further Research}

The primary purpose of further research is to improve the application level of inclusive design for diverse users and complex contexts, for example, to provide support for comprehensive human factors design research of a specific product in diversified usages. On the one hand, the research goal lies in the boundary of inclusive solutions expanded by the creativity of artificial design; on the other hand, machine learning can significantly improve the design efficiency of using existing knowledge to respond to differentiated demands.

According to the above research objectives, an ideal research environment should enable the establishment of interdisciplinary teams and platforms so that design research can be closely combined with relevant cutting-edge technology research, and an active division of task force can be carried out. Inclusive design research mainly focuses on exploring the technical, humanistic, and social reasons behind the existing problems and challenges, exploring the potential diverse needs in different contexts, and seeking strategies, methods, and tools to solve the problems, overcome the challenges and meet the needs. It means that designers need to create more design patterns that can be driven by algorithms. However, scientific and technological research provides more in-depth understandings, more effective technical means, and more possibilities for how to solve the problem of inclusiveness and realize these design patterns. Corresponding design practices can serve the technology transfer and commercialization of different scientific research outcomes, expand a broader range of application fields for artificial intelligence technology besides improving performance, and capture potential business opportunities.

\section{Discussion}

An inclusive design research from Cambridge University shows that there is an excellent potential for adaptation between AI-based Advanced Driver Assistance Systems and the diversity of drivers [16]. Some researchers try to introduce AI support to codesign [17], while others choose to apply inclusive design approaches to the development of AI products [18]. In the interdisciplinary research environment, we suggest that it may be necessary to focus on the application of machine learning in the design process. Firstly, through the establishment of a machine learning system with an interface for sensing user needs/capabilities and context features, the evaluation criteria and requirements of the target test set are studied so that machine learning can identify the design requirements and design problems corresponding to user and context features under a given design purpose. Secondly, together with researchers of artificial intelligence, the training models and feature representations of machine learning are optimized for different scenarios and tasks. Inclusive design tools, processes, and methods combined with machine learning technology are studied so that machine learning can identify corresponding design patterns or design options after inputting changing design requirements and problems. After reaching the milestone of phased research, new design tools, processes, and methods can be put into applied research in different subareas to summarize and establish corresponding domain knowledge and design pattern libraries. The outcomes of further research will provide reference for understanding 
inclusive design patterns applicable to different specific fields and the principles and mechanisms behind them, and improve the method of algorithmically providing appropriate design responses to a specific need.

\section{References}

1. Bianchin, M., Heylighen, A.: Fair by design. Addressing the paradox of inclusive design approaches. Design Journal 20, S3162-S3170 (2017)

2. Bianchin, M., Heylighen, A.: Just design. Design Studies 54, 1-22 (2018)

3. Li, F., Dong, H.: The Economic Explanation of Inclusive Design in Different Stages of Product Life Time. Proceedings of the Design Society: International Conference on Engineering Design 1, 2377-2386 (2019)

4. Li, F., Dong, H.: Achieving Inclusion with Contextualized User-Sensitive Design. In: Antona, M., Stephanidis, C. (eds.) 2019. LNCS, vol. 11572, pp. 113-132. Springer, Orlando (2019)

5. Design Council (UK), https://www.designcouncil.org.uk/news-opinion/what-frameworkinnovation-design-councils-evolved-double-diamond

6. Lake, B.M., Salakhutdinov, R., Tenenbaum, J.B.: Human-level concept learning through probabilistic program induction. Science 350, 1332-1338 (2015)

7. Marcus, G.: Deep Learning: A Critical Appraisal. arXiv e-prints, (2018)

8. Huang, S., Papernot, N., Goodfellow, I., Duan, Y., Abbeel, P.: Adversarial Attacks on Neural Network Policies. arXiv e-prints, (2017)

9. Goodfellow, I., Bengio, Y., Courville, A.: Deep Learning. The MIT Press, Cambridge, MA (2016)

10.Lake, B., Baroni, M.: Still not systematic after all these years: On the compositional skills of sequence-to-sequence recurrent networks. 6th International Conference on Learning Representations, Vancouver, BC, Canada (2018)

11.Samek, W., Wiegand, T., Müller, K.-R.: Explainable Artificial Intelligence: Understanding, Visualizing and Interpreting Deep Learning Models. arXiv e-prints, (2017)

12.Radford, A., Metz, L., Chintala, S.: Unsupervised Representation Learning with Deep Convolutional Generative Adversarial Networks. arXiv e-prints, (2015)

13.Gheshlaghi Azar, M., Piot, B., Avila Pires, B., Grill, J.-B., Altché, F., Munos, R.: World Discovery Models. arXiv e-prints, (2019)

14.Liu, Z., Miao, Z., Zhan, X., Wang, J., Gong, B., Yu, S.X.: Large-Scale Long-Tailed Recognition in an Open World. IEEE Conference on Computer Vision and Pattern Recognition, pp. 2537-2546. Computer Vision Foundation, Long Beach, CA, USA (2019)

15.Dong, H., Nickpour, F., McGinley, C.: Designing Ergonomic Data Tools for Designers. In: ICED 09 - the 17th International Conference on Engineering Design, pp. 53-63. (2009)

16.Caber, N., Langdon, P., Clarkson, P.J.: Designing Adaptation in Cars: An Exploratory Survey on Drivers' Usage of ADAS and Car Adaptations. In: Stanton, N. (ed.) AHFE International Conference on Human Factors in Transportation, 2019, vol. 964, pp. 95-106. Springer Verlag (2020)

17.Walsh, G., Wronsky, E.: AI + co-design: Developing a novel computer-supported approach to inclusive design. In: 22nd ACM Conference on Computer-Supported Cooperative Work and Social Computing, CSCW 2019, pp. 408-412. Association for Computing Machinery, (2019) 18.Spencer, D.J., Poggi, J., Gheerawo, R.: Designing out stereotypes in artificial intelligence: Involving users in the personality design of a digital assistant. In: 4th EAI International Conference on Smart Objects and Technologies for Social Good, GOODTECHS 2018, pp. 130135. Association for Computing Machinery, (2018) 